\title{
A Transmission Line Modeling Program Written in C
}

\author{
DAVID G. MESSERSCHMITT, FELLOW, IEEE
}

\begin{abstract}
LINEMOD is a transmission line modeling program that takes a line configuration (including possibly bridged taps) and generates the input impedance, attenuation versus frequency, and impulse response. It is written in $\mathrm{C}$, a modern structured programming language, and utilizes sophisticated data structures, dynamic storage allocation, and recursion to achieve flexibility and economy of storage. As such, it illustrates the value of $\mathbf{C}$ as an implementation language for modeling programs of this type. It also emphasizes a shortcoming of $\mathrm{C}$ for this purpose, which is the absence of built-in complex arithmetic.
\end{abstract}

\section{INTRODUCTION}

L INEMOD is a program that models transmission lines containing (possibly) different gauges of wire, bridged taps, resistive sources, shunt resistor-capacitor terminations, and transformers. Other types of circuit elements can be added easily. It is implemented in $\mathrm{C}$ language [1], a representative modern structured language with features such as sophisticated data structures, dynamic allocation of memory, and recursion (another notable example would be Pascal). Thus, LINEMOD serves to illustrate the value of modern structured programming techniques [3] as applied to a communications system modeling problem.

There are several aspects to the methodology of modern structured programming. The most important one is the thoughtful partitioning of the program, with carefully defined interfaces between the pieces. This aspect is largely independent of the programming language used. A second aspect is the availability and use of structured control-flow statements such as "if-else" and "while" that a language like $\mathrm{C}$ or Pascal provides, making the code more readable and understandable. Here again this facility can be provided in a Fortran environment by using a preprocessor such as RATFOR [2], and the latest versions of Fortran also provide these control statements. A third aspect is the availability of powerful facilities like user-defined data types and structures, dynamic allocation of memory, and recursion. These facilities are not provided in Fortran (except for a simple form of dynamic memory allocation in recent version), and LINEMOD illustrates how these facilities can be used to advantage in a communications model-

Manuscript received August 15, 1983; revised September 10, 1983. This work was supported by grants from Racal-Vadic, Advanced Micro Devices, Fairchild Semiconductor, Harris Semiconductor and Digital Telephone Systems, and National Semiconductor, with a matching grant from the University of California Microelectronics and Computer Opportunities Research Program.

The author is with the Department of Electrical Engineering and Computer Science, University of California, Berkeley, CA 94720. ing program to make the program more flexible in its use and simpler to write and understand.

LINEMOD also reveals a major weakness of the $\mathrm{C}$ language for this type of program; namely, the absence of complex number arithmetic. This weakness is easily overcome because of the ability of the programmer to define new data types, such as complex numbers, but this is somewhat cumbersome when compared to a language like Fortran with built-in complex arithmetic.

In summary, the emphasis of this paper is on illustrating the power that a modern structured language like $\mathrm{C}$ can bring to a communication system modeling program, and at the same time illustrates some weaknesses of such a language for this task. It is hoped that this illustration will help other programmers in the choice of an implementation language.

\section{Description OF LINEMOD}

This section will describe the LINEMOD program: its function and user interface, as well as the modeling techniques used.

LINEMOD was written because of the need for a program to model transmission lines in a project to design full-duplex data modems operating over subscriber loops. It has been used in conjunction with BLOSIM [5] to provide a model for a transmission line in a functional simulation of the modem. A major feature of subscriber loops, in addition to uniform transmission lines, is the presence of bridged taps (open circuited transmission lines in parallel with the main transmission line), and other circuit elements such as resistors, capacitors, and transformers. In addition, it was desired to model arbitrary filter transfer functions in cascade with the line.

The input to LINEMOD is a file containing the specification of the circuit elements in the line in the order in which they appear. One important element is a bridged tap, the circuit elements of which must then be specified. Bridged taps can themselves contain any elements, including more bridged taps.

The output of LINEMOD presently consists of three files, one containing the input impedance of the line versus frequency, another containing the frequency response of the line (magnitude of the voltage transfer function in decibels), and the third including the impulse response. A user supplied routine can supply a transfer function that is 
placed in tandem with the line, thereby permitting the modeling of tandem filters (such as transmit and receive filters) and input pulses other than impulses.

LINEMOD is used in conjunction with the functional simulation tool BLOSIM [5] in the following manner: For a given physical configuration of a transmission line together with filters, transformer, etc., LINEMOD outputs the files enumerated above. The impulse response file in particular is read by a BLOSIM STAR routine which models the line with this impulse response. LINEMOD is not incorporated directly into the BLOSIM simulation because typically many BLOSIM simulations are performed for a given transmission line configuration.

A standard technique for mathematically representing the line was used, the ABCD matrix [4]. An ABCD matrix is a two-by-two matrix with complex elements that relates at a particular frequency the input voltage and current to the output voltage and current for a twoport. It has the desirable feature that the $A B C D$ matrix of a cascade of a pair of twoports is the product of the $A B C D$ matrices of the individual twoports.

In LINEMOD, the line is considered to be a cascade of twoports, where a bridged tap is represented as a shunt impedance with value equal to the input impedance of the bridged line. Internally, LINEMOD calculates the ABCD matrix of the line by multiplying the $\mathrm{ABCD}$ matrices of each twoport in the line.

The ABCD matrix of a uniform transmission line was obtained from a polynomial approximation to the primary parameters of the line (resistance, inductance, capacitance, and admittance per unit length).

From the user point of view, the transmission line is specified by an input file that has a simple but very flexible syntax. Each line section, bridged tap, resistor, capacitor, etc., is represented by one line in the file. In addition, there is a first line that specifies certain parameters controlling the spacing of points in the time and frequency response.

The syntax of the input file will be illustrated by example. The following file specifies an $18 \mathrm{~km}$ line (24 gauge) with a $5 \mathrm{~km}$ bridged tap in the center. The bridged line is not terminated (open circuited). The source and termination resistors are $100 \Omega$.

\section{1.25e-5 \# Time and frequency spacing information}

\# Main line

series_r 100.

line 924

\# Bridged tap
bridge
line 524
end

\# Back to the main line

line 924

shunt_r 100.

end

As a second example, just for fun, terminate the bridged tap in $5 \mathrm{k} \Omega$ and add a bridged tap to the center of the bridged. tap:

\section{$531.25 \mathrm{e}-5$}

\# Main line

series_r 100.

line 924

$$
\begin{aligned}
& \text { \# Bridged tap } \\
& \text { bridge } \\
& \text { line } 2.524 \\
& \text { \# This bridged tap has its own bridged tap } \\
& \text { bridge } \\
& \text { line } 0.524 \\
& \text { end }
\end{aligned}
$$

line 2.524

shunt_r 5000 .

end

\# Back to the main line

line 924

shunt_r 100.

end

This illustrates that the bridged taps can be nested an arbitrary number of times; that is; bridged taps can have bridged taps can have bridged taps, etc. While this was not an essential feature for the program, it turns out to come for free in the implementation approach described below.

The implementation described below was specifically designed to minimize the effort in adding new models. As an illustration, a transformer model was recently added with a total programming effort of only about 10 minutes! An additional objective was to be able to add new output files with equal ease. The philosophy of the program was to build a tool that would be flexible and of lasting value.

\section{IMPLEMENTATION}

This section describes the implementation of LINEMOD. Particular emphasis is given to the areas where modern features of the $\mathrm{C}$ language were very helpful, and conversely, where shortcomings of the language were evident. In this discussion, it will not be assumed that the reader is familiar with the language.

\section{A. Overall Structure}

The overall structure of the program is straightforward, and illustrated in Fig. 1. First the input file is read and interpreted, and the data structure containing the topology and parameters of the line is built. The next step calculates the ABCD matrix at each frequency of interest, and builds a data structure containing this information. The final step is to generate the output files. Each of these steps will now be examined in more detail.

\section{B. Reading the Input File}

The format of the input file is the primary user interface, and should be designed with care. Each line in the input 


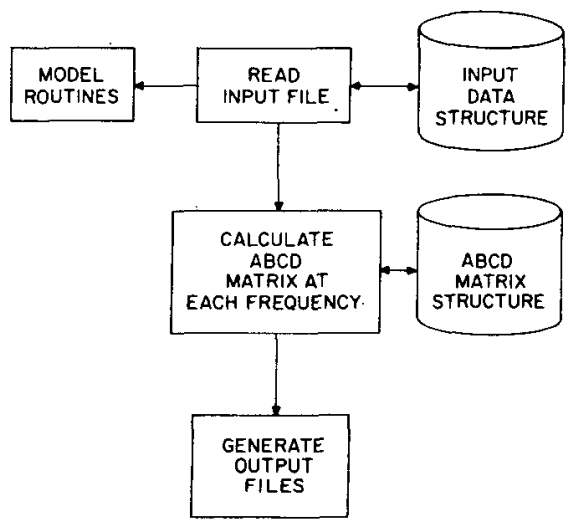

Fig. 1. Overall structure of LINEMOD.

file corresponds to a twoport in the transmission line model. Each line must therefore be interpreted as to the type of twoport and its parameters, and this information must be stored for future reference. LINEMOD depends on a separate model routine to both read an input line and provide the parameters of an ABCD matrix for the corresponding twoport. While this may seem to be a strange partitioning, the advantage is that it becomes straightforward to add new twoport models. All the information about a model is embedded in a single routine, and adding a model does not impact the remainder of LINEMOD at all. The way it works is that LINEMOD reads the first word of each input line, and calls a routine by the same name that must be provided in the library of model routines. This routine is passed the input line, and has the responsibility of reading the remainder of the line and storing the parameters in permanent memory in its own input data structure.

It is the area of data structures where the power of $\mathrm{C}$ begins to be evident. Flexibility must be maintained in both the variety of models that must be accommodated, as well as in the total number of twoports that must be accommodated in a given line. With respect to the former problem, $\mathrm{C}$ enables the programmer to define new data types, and in LINEMOD a new type is defined for each twoport model. For example, here is the definition of the data type TRANSFORMER, which stores the parameters of a transformer (inductance and coupling coefficient):

typedef struct \{

float inductance; $/{ }^{*}$ winding inductance* $/$

float $\mathrm{k} ; \quad /{ }^{*}$ coupling coefficient $* /$

\section{\} TRANSFORMER;}

This C "structure" can be used to create one section of memory that contains all the parameters of a transformer twoport. Whenever a transformer is encountered in the input file, the modeling routine can allocate memory dynamically to store the parameters of the transformer. The statements required to do this are

TRANSFORMER $*$;

$p=\operatorname{calloc}(1, \operatorname{sizeof}($ TRANSFORMER $)$;
First, variable $p$ is declared to be a "pointer" to a data object of type TRANSFORMER; that is, $p$ is the address of this data object in memory. The $\mathrm{C}$ unary operator sizeof( ) returns the size of the data object TRANSFORMER in bytes, and calloc( ) calls the operating system to dynamically allocate a block of memory of this size and return a pointer to this memory, which is assigned to $p$. Subsequently, when this modeling routine is asked to provide the $\mathrm{ABCD}$ matrix for this twoport, it is passed a pointer to this structure, which it consults to get the parameters of the twoport.

Observe that exactly the right amount of memory is allocated at run time for storing the parameters of the line specified by the user. No assumption has to be made ahead of time as to the type or number of twoports, and no extra memory is required (which would slow the execution of the program because of the requirement for extra paging).

Similarly, as the input file is being read, LINEMOD itself dynamically creates a data structure that contains the configuration of the line. Fig. 2 illustrates how these data are stored. Fig. 2(a) shows a typical transmission line configuration, with a main line (labeled "in" and "out"), and two bridged taps (one of which has its own bridged tap). Fig. 2(b) illustrates how this transmission line is represented in memory, namely, as a tree of C structures, each node of the tree containing the location of the type and parameter information for the twoport, as well as the information on how that twoport is connected to the remainder of the line. Finally, Fig. 2(c) shows explicitly the data structure corresponding to a twoport in the line, which consists of four pointer variables. The first pointer is the address of the modeling routine that can provide the ABCD matrix for this twoport (which implicitly gives the type of twoport), a pointer to the parameters of this twoport (such as the TRANSFORMER data structure defined earlier), a pointer to the data structure for the next twoport in the main transmission line, and finally a pointer to the first twoport in any bridged tap connected to the line at that point. If the twoport is the last in the line or there is no bridge tap, the corresponding pointer is NULL (having value zero, indicating that the data structure does not exist).

Notice that this data structure has absolutely no information embedded in it as to the model itself, but rather that information is embedded in the model routine and the data structure that only it creates and accesses. Note also that this data structure can accommodate any number of twoports in the line and any tree-like line topology (including bridged taps with their own bridged taps, etc.) and uses exactly the amount of memory required to represent that line (the memory for the data structure of Fig. 2(c) is created dynamically as needed as the input file is read).

Another very powerful feature of $\mathrm{C}$ is recursion; that is, routines can call themselves. This neatly solves one problem encountered in reading the input file, that is, how to recognize the end of a bridged tap, and where to return in the main line at the end of a bridged tap. A flow diagram of the routine read_file( ), which reads lines from the input 


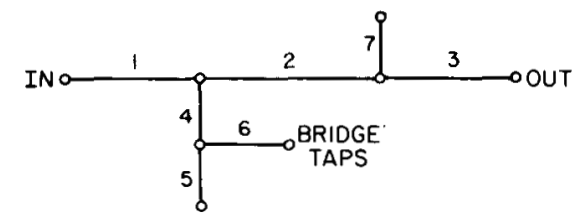

(a)

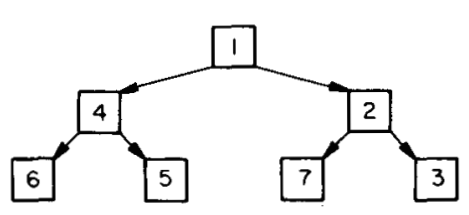

(b)

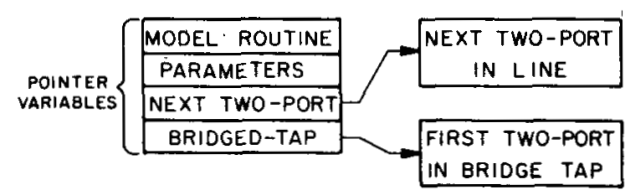

(c)

Fig. 2. Data structure for the transmission line. (a) Topology of a line with bridge taps. (b) Line topology as a tree. (c) Data structure for each twoport.

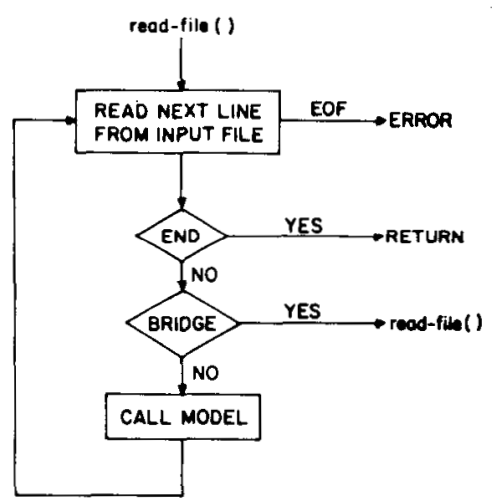

Fig. 3. Flow diagram of read_file ( ).

file until it encounters a line "end," is shown in Fig. 3. Note that read_file( ) does not necessarily read the whole file, but only the lines up to the first matching "end." It starts by reading one line from the file, and if that line is not available (indicated by an end-of-file EOF) there is an error because an "end" has not been encountered. If that line is an "end," then the routine has completed its job and can return.

If the line is "bridge," that indicates the beginning of a bridge tap. It is at this point that recursion is very handy, since the routine can simply call itself to read in the data on the parameters of this bridged tap from the same input file. No special logic is required to handle this case, since the new call to read_file( ) simply continues reading the file until it encounters the "end" marking the end of the specification of the bridged tap, and then returns to the original read_file( ) to continue reading the specification of the main line. Note that recursion has made this approach at the same time very flexible and trivial to program. It also neatly handles the case where bridged taps

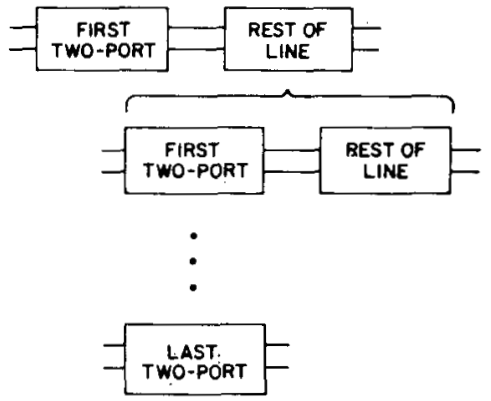

Fig. 4. Recursive definition of twoports in line.

themselves have bridged taps through two or more levels of recursion.

Finally, if the input line is neither "end" or "bridge," it corresponds to a twoport, and the corresponding modeling routine is called to complete the job of interpreting the line and creating the data structure.

\section{Calculating the ABCD Matrix}

The $A B C D$ matrix for each section of the line is calculated by the modeling routine. The ABCD matrix for the entire line is calculated by the routine abcd( ). As illustrated below, the recursive feature of $\mathrm{C}$ again greatly simplifies the logic of the program.

Ignoring the possibility of bridged taps for a moment, the use of recursion is illustrated in Fig. 4. The line can be divided into the first twoport and the remainder of the line. The ABCD matrix of the first twoport can be calculated, the $A B C D$ matrix of the rest of the line can be calculated, and the overall $A B C D$ matrix can be determined by multiplying these two matrices. The method of calculating the $\mathrm{ABCD}$ matrix of the rest of the line is, as illustrated, to divide it into the first twoport and the remainder. The calculation proceeds recursively until the last twoport in the line is encountered.

The flow diagram for abcd( ), which also takes into account bridged taps, is shown in Fig. 5. The first step is to calculate the $A B C D$ matrix for the first twoport in the line. If this twoport is not a bridged tap, the appropriate modeling routine is simply called to return its $A B C D$ matrix (note again the independence from the type of twoport). If it is a bridged tap, abcd( ) is first called recursively to determine the $A B C D$ matrix representation for the bridged tap line, from which the input impedance can be inferred.

Once the $A B C D$ matrix of the first twoport has been determined, the final step is to determine the ABCD matrix of the rest of the line. If the original twoport was the last in the line, then we are done. Otherwise, abcd( ) is again called recursively to determine the $\mathrm{ABCD}$ matrix of the rest of the line, and the two $A B C D$ matrices that have been determined are multiplied.

The result of the $A B C D$ matrix calculation is a data structure consisting of an array of $\mathrm{ABCD}$ matrices, one at each frequency of interest (again this is a data structure that is easy to create in C). 


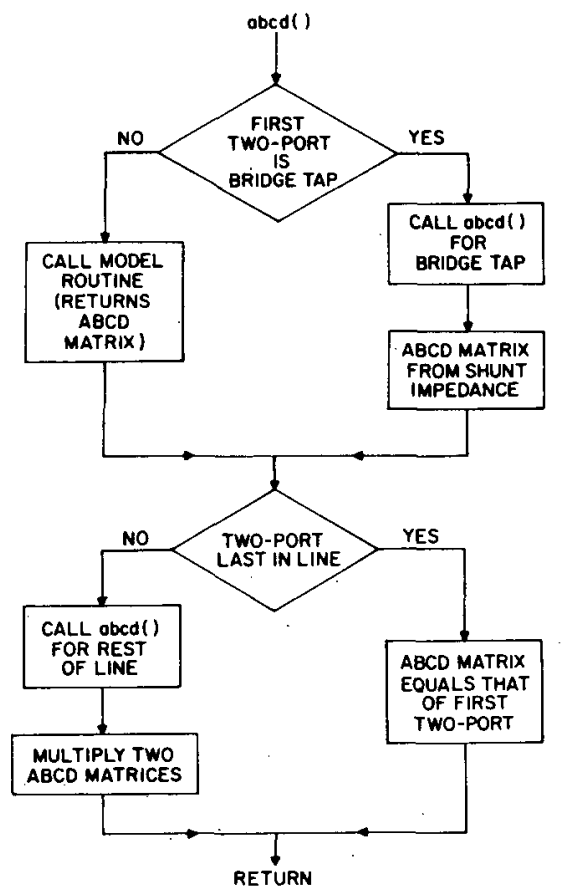

Fig. 5. Flow diagram of abed( ) to calculate ABCD matrix.

The ABCD matrix has complex valued elements, and the $C$ language has no built-in complex arithmetic capability. This implies that the programmer has to provide his own complex number arithmetic, and this is a substantial disadvantage. The way this was done in LINEMOD was to define a complex number structure

typedef struct \{

float real; $/{ }^{*}$ real part*/

float imag; $/{ }^{*}$ imaginary part*/

\section{\} COMPLEX;}

and then write a set of routines that implement the complex arithmetic functions such as add, multiply, modulus, etc. For example, here is a complex multiply routine:

COMPLEX cmpy $(a, b)$

COMPLEX $a, b$;

\{

\section{COMPLEX $c$;}

$c$. real $=a$. real ${ }^{*} b$. real $-a$. imag $^{*} b$. imag;

$c$. imag $=a \cdot$ real ${ }^{*} b$. imag $+a$. imag. ${ }^{*} b$. real;

return(c);

\}

While this is simple and need only be done once, all complex arithmetic must still be done with function calls, which is execution-time consuming, and makes the code more difficult to write and read. For example, the statement required to reproduce the Fortran expression

$$
\text { COMPLEX } a, b, c
$$$$
\mathrm{c}=a^{*} a^{*} b
$$

in $\mathrm{C}$ is, using this approach:

$$
\begin{aligned}
& \text { COMPLEX } a, b, c ; \\
& c=\operatorname{cmpy}(\operatorname{cmpy}(a, a), b) ;
\end{aligned}
$$

A more computationally efficient approach would be to define macros for the complex arithmetic operations and then use the macro preprocessor of the $\mathrm{C}$ compiler, but this is even more cumbersome to use. For example, here is how the Fortran expression

$$
\begin{aligned}
& \text { COMPLEX } a, b, c ; \\
& c=a^{*} b
\end{aligned}
$$

would be reproduced in $\mathrm{C}$ using this approach:

$$
\begin{aligned}
& \text { \# define } \operatorname{rcmpy}(A, B) A \cdot \operatorname{real}^{*} B \cdot \operatorname{real}-A \cdot \operatorname{imag}^{*} B \cdot \operatorname{imag} \\
& \text { \# define } \operatorname{icmpy}(A, B) A \cdot \operatorname{real}^{*} B \cdot \operatorname{imag}+A \cdot \operatorname{imag}^{*} B \cdot \text { real } \\
& \text { COMPLEX } a, b, c ; \\
& c . r e a l=\operatorname{rcmpy}(a, b) ; \\
& c . \text { imag }=\operatorname{icmpy}(a, b) ;
\end{aligned}
$$

The difficulty here is that the real and imaginary parts of the result of the multiplication must be separately defined as macros. The efficiency results, however, from the fact that the code for the details of the multiplication are substituted into the program by the macro preprocessor prior to compilation, and function calls for each complex arithmetic operation are thereby avoided.

\section{Generating the Output Files}

Once the array of $\mathrm{ABCD}$ matrices is available, it is easy to calculate any quantity of interest, such as the input impedance of the line or the voltage transfer function. The three output files are generated by three separate routines that access this common data structure. It would be simple to add other routines to generate output files that access this same data structure.

\section{CONCLUSIONS}

LINEMOD has illustrated how the modern features of the $\mathrm{C}$ language, such as user defined data types, data structures, dynamic memory allocation, and recursion, can be used to advantage in the development of a communications modeling program. The result is a program with great flexibility and generality, which can accommodate small or large systems without squandering memory resources, and yet with little additional programming effort. The structured design of LINEMOD which enables added features and changes to be made easily could certainly be reproduced in another language such as Fortran, although with greater difficulty because of the lack of the powerful userdefined data structures. It is also evident how the use of recursion made the program very flexible and at the same time logically simple. 
On the other hand, the absence of complex arithmetic as an explicit part of the language is a definite disadvantage of the $\mathrm{C}$ language when applied to communications modeling tasks.

\section{ACKNOWLEDGMENT}

The author is indebted to J. Gorecki of Intel Corporation for his assistance in developing the models for the primary parameters of a uniform transmission line.

\section{REFERENCES}

[1] B. W. Kernighan and D. M. Ritchie, The C Programming Language Englewood Cliffs, NJ: Prentice-Hall, 1978.

[2] - Software Tools. Reading, MA: Addison-Wesley, 1976.

[3] O.-J. Dahl, E. W. Dijkstra, and C. A. R. Hoare, Structured Programming. New York: Academic, 1972.

[4] S. V. Ahamed, "Simulation and design studies of digital subscriber lines," Bell Syst. Tech. J.; vol. 61, July-Aug. 1982

[5] D. G. Messerschmitt, "A tool for structured functional simulation," this issue, pp. 137-147.

David G. Messerschmitt (S'65-M'68-SM'78-F'83), for a photograph and biography, see this issue, p. 147.

\title{
Techniques for Estimating the Bit Error Rate in the Simulation of Digital Communication Systems
}

\author{
MICHEL C. JERUCHIM, SENIOR MEMBER, IEEE
}

\begin{abstract}
Computer simulation is often used to estimate the bit error rate (BER) performance of digital communication systems. There are a number of distinct techniques in the simulation context that can be used to construct this estimate. A tutorial exposition of such techniques is provided, with particular reference to five specific methods which can be implemented in a simulation. These methods range from the traditional Monte Carlo trials to assumption of definite forms for the noise statistics. An attempt is made to show how these methods are related, and the specific assumptions that are invoked in order to apply them.
\end{abstract}

\section{INTRODUCTION}

$\mathrm{O}$ VER the past decade or so, it has become increasingly common to resort to computer-aided techniques to estimate the performance of digital communication systems. This trend has been particularly apparent in satellite design, largely because of the difficulty of analyzing in closed form a band-limited nonlinear system, which in general characterizes a digital satellite link. The definition of performance almost universally used is the bit error rate (BER) or the bit error probability. There are a number of ways to arrive at an estimate of the BER, each with its own advantages and disadvantages. Here we use the term

Manuscript received May 5, 1982; revised May 23, 1983.

The author is with the Space Systems Division, General Electric Company, Philadelphia, PA 19101. "estimate" with a broader connotation than its strictly statistical sense, to signify a method of computation which results in a number that may not be interpretable as a classical estimate. The following discussion, while initially motivated by the satellite application, is in principle applicable to terrestrial microwave systems, voiceband data communications, or any context where the BER is the performance criterion.

The applicability or usefulness of a given estimation method does depend in general on the particulars of the modulation/coding scheme and associated receiver structure. Although it is outside our intended scope to deal with these particulars directly, we do mention that all of the methods we shall consider are applicable to, or were originally conceived in connection with, conventional systems, i.e., systems where decisions are made on a bit-by-bit basis by observing (sampling or integrating) a baseband waveform within each bit interval and comparing the observation against the decision regions (threshold sensing). This is our basic context, which will be implicit throughout, although for clarity of exposition we shall usually deal explicitly with either a single binary channel (e.g., BPSK), which is the simplest and most easily visualizable situation, or its natural extension, two independent binary channels (e.g., QPSK). Later, in Section VII, we shall comment on 